\title{
A Brief Review of Some Local Approaches for the Failure Assessment of Brittle and Quasi-Brittle Materials
}

\author{
F. Berto \\ Department of Management and Engineering, University of Padua, Stradella San Nicola 3, 36100 Vicenza, Italy \\ Correspondence should be addressed to F. Berto; filippo.berto@unipd.it
}

Received 14 November 2013; Accepted 14 January 2014; Published 25 February 2014

Academic Editor: Manuel Elices

Copyright (C) 2014 F. Berto. This is an open access article distributed under the Creative Commons Attribution License, which permits unrestricted use, distribution, and reproduction in any medium, provided the original work is properly cited.

\begin{abstract}
Brittle failure of components weakened by cracks and notches is a topic of active and continuous research. It is a key topic for all researchers who face the problem of fracture of materials under different loading conditions and deals with a large number of applications in different engineering fields. This topic is significant in all the cases where intrinsic defects of materials or geometrical discontinuities give rise to localized stress concentration which, in brittle materials, may generate a crack leading to catastrophic failure or to a shortening of the assessed structural life. Whereas cracks are viewed as unpleasant entities in most engineering materials, U- and V-notches of different acuities are sometimes deliberately introduced in design and manufacturing of structural components. The main aim of the present contribution is to present a short review of some local approaches applicable near stress raisers both sharp and blunt allowing the reader to have an update state of the art for the considered criteria.
\end{abstract}

\section{Introduction}

Brittle failure of components in particular when weakened by cracks and sharp and blunt $\mathrm{V}$-notches is a relevant topic of active and continuous research. It is attractive for all researchers who face the problem of fracture of materials under different loading conditions and deals with a large number of applications in different engineering fields, not only with the mechanical one. It is no longer uncommon for the scientists and engineers to speak of the same language when dealing with the design and manufacturing of intricate devices and this is particularly true when they are nanometer or smaller in size and large-scale effects take place. In fact, the attempt to strike a common ground on which physical events might be connected is a curiosity that arises naturally in research. By analyzing the topography of the cracking of ice-wedge polygons in Arctic permafrost of mud flats in Death Valley and of craze-cracks (heat-checking) at the bore of a gun tube it is easy to observe that they are all strikingly similar. However, they span five orders of magnitude in scale, with the maximum plate dimensions for ice and mud being, respectively, 22 and 0.25 meters and with the minimum plate size for gun tube craze cracking being about 0.2 millimeters.
Design based on damage tolerance criteria often deals with notched components giving rise to localized stress concentrations which, in brittle materials, may generate a crack leading to catastrophic failure or to a shortening of the assessed structural life.

When the stress concentrators are cracks, or sharp Vnotches, the tools derived from linear elastic fracture mechanics are applicable. As soon as the notch is blunted, that is, the notch root radius is not zero, the stress singularity disappears and linear elastic fracture mechanics is no longer applicable. The problem becomes more involved if the loading symmetry is lost, that is, when the notched structural component is subjected to mixed mode loading. Moreover modelling damage around blunted notches has proven very difficult and is strongly dependent on the microstructural aspects of each material. In fact, under mixed mode loading, particularly for notches with a nonnegligible radius, providing a suitable unifying fracture criterion is the current challenge which requires further investigations. The scarcity of experimental results available in the literature dealing with blunt notches under prevalent mode II, mode III, or compression loadings confirms that the problem of brittle or quasi-brittle fracture of blunt notched components is far from being completely solved. 
The most important and revolutionary concept for the fracture assessment is that based on the definition of an "elementary" volume and "structural support length" introduced many years ago by Neuber [1-3]. It states that not the theoretical maximum notch stress is the static or fatigue strengtheffective parameter in the case of pointed or sharp notches, but rather the notch stress averaged over a short distance normal to the notch edge. The concept can be applied not only to static loadings but also to high cycle fatigue regime. As an extension of Neuber's concept, more recently Radaj proposed the application of the fictitious notch rounding for the fracture and fatigue assessment of structural materials [4-6].

Fundamentals of critical distance mechanics applied to static brittle failure state that crack propagation occurs when the normal strain or circumferential stress at some critical distance from the crack tip reaches a given critical value. The proposal of mode I dominance for cracked plates which is another key concept was suggested by Erdogan and Sih in their pioneering work dated 1963 [7]. It deals with cracked plates under plane loading and transverse shear and states that the crack grows in the direction almost perpendicular to the maximum tangential stress in radial direction from its tip. Some recent and effective improvements of the maximum tangential stress criterion have been proposed by Ayatollahi and coworkers [8-12] taking into account also the contribution due to the T-stress. Other researchers applied the Point and Line methods to assess the fatigue and fracture behaviour of different materials weakened by sharp and blunt notches [13-17].

Dealing with the strain energy density concept, it is worthwhile contemplating some fundamental contributions by Sih where the concept of "core region" surrounding the crack tip was proposed. The main idea is that the continuum mechanics stops short at a distance from the crack tip, providing the concept of the radius of the core region. The strain energy density factor $(S)$ was defined as the product of the strain energy density by a critical distance from the point of singularity. Sih's criterion is a point-wise criterion. Failure was thought of as controlled by a critical value of the $S$-factor, whereas the direction of crack propagation was determined by imposing a minimum condition on $S$ [18-20].

Gillemot, first, measured the deformation energy required for crack initiation in a unit volume of material calling that parameter Absorbed Specific Fracture Energy (ASFE). A simple link between the strain energy density factor $(S)$ and the ASFE was found [21-23]. Considering the fracture energy some interesting developments have been carried out by Elices and coworkers dealing with the cohesive zone model applied to different materials and weakened not only by cracks but also by notches [24-28].

Different from Sih's criterion, which is a pointwise criterion, the averaged strain energy density criterion has been proposed in [29] and applied combining the concept of elementary volume proposed by Neuber and the local mode I concept proposed by Erdogan and Sih. The new criterion states that brittle failure occurs when the mean value of the strain energy density over a control volume (which becomes an area in two dimensional cases) is equal to a critical energy for the material. The SED approach is based both on a precise definition of the control volume and the fact that the critical energy does not depend on the notch sharpness. Such a method was formalised and applied first to sharp, zero radius, $\mathrm{V}$-notches and later extended to blunt $\mathrm{U}$ - and V-notches under mode I loading [30] and applied to welded joints [31-33]. The control radius of the volume, over which the energy has to be averaged, depends on the ultimate tensile strength, the fracture toughness, and Poisson's ratio in the case of static loads, whereas it depends on the unnotched specimen's fatigue limit, the threshold stress intensity factor range, and Poisson's ratio under high cycle fatigue loads. The approach was successfully used under both static and fatigue loading conditions to assess the strength of notched and welded structures subjected to predominant mode I and also to mixed mode loading. Dealing with both notched and welded components, a final synthesis of more than 2400 experimental data from static and fatigue tests has been carried out. Very different materials have been considered with a control radius, ranging from $0.4 \mu \mathrm{m}$ to $500 \mu \mathrm{m}[34,35]$.

Dealing with brittle failure of notched components and summarising the most recent experimental results reported in the literature, a complete review of the main local approaches applicable near stress raisers both sharp and blunt has been carried out in [35]. Among the discussed criteria attention is paid to the volume-based SED approach developed in the above mentioned paper and recently applied to assess the brittle failure of a large bulk of materials. After an accurate summary of the analytical frame and the theoretical basis, some recent data from isostatic graphite (see [36] and references therein), largely used atomic reactors for excellent isotropic electrical, thermal, and mechanical properties, under different loading conditions have been discussed in detail together with data from polymethyl methacrylate [37]. A final synthesis summarising all the data available up to now from very different materials is reported in the paper. The application of the SED approach to three-dimensional problems and the advantages of the approach in comparison with other proposed local approaches are discussed in [35] as well.

A short review of some local approaches familiar to the author and applicable near stress raisers both sharp and blunt is reported in the present paper. Recent useful references are provided for authors engaged in such topics giving an update state of the art of each considered criterion.

\section{Generalized Maximum Tangential Stress Criterion}

One of the fundamental idea in the fracture assessment of brittle fracture is the so-called local mode I concept. The proposal of mode I dominance was suggested in [7] dealing with cracked plates (see Figure 1) under plane loading and transverse shear, where the crack grows in the direction almost perpendicular to the maximum tangential stress (MTS) in radial direction from its tip. This theory is one of the widely used theories for mixed mode crack growth. In more detail the criterion states that the crack propagation starts along the direction on which the tangential stress becomes maximum. Along that line the shear stress is equal to zero 


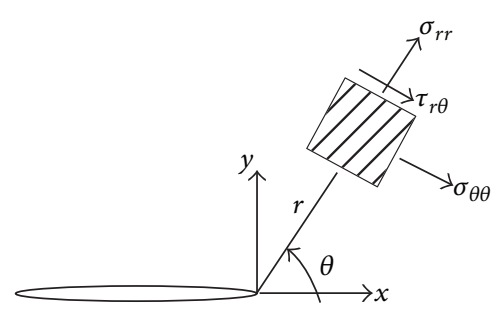

FIGURE 1: Plate with a central crack.

and a principal stress state occurs. The fracture starts when the maximum tangential stress $\left(\sigma_{\theta}\right)$ reaches a critical stress $\left(\sigma_{c}\right)$ for the material equal to the fracture stress in uniaxial tension. Mathematically the conditions for the crack growth direction can be expressed by the following equations:

$$
\begin{gathered}
\frac{\partial \sigma_{\theta \theta}}{\partial \theta}=0, \quad \frac{\partial^{2} \sigma_{\theta \theta}}{\partial \theta^{2}}<0, \\
\sigma_{\theta \theta}\left(\theta_{c}\right)=\sigma_{c} .
\end{gathered}
$$

The singular stress field in the vicinity of the crack tip is expressed by the following equation:

$$
\begin{aligned}
& \left\{\begin{array}{l}
\sigma_{\theta \theta} \\
\sigma_{r r} \\
\tau_{r \theta}
\end{array}\right\} \\
& =\frac{K_{I}}{4 \sqrt{2 \pi r}} \times\left[\left(\begin{array}{c}
3 \cos \left(\frac{\theta}{2}\right) \\
5 \cos \left(\frac{\theta}{2}\right) \\
\sin \left(\frac{\theta}{2}\right)
\end{array}\right)+\left(\begin{array}{c}
\cos \left(\frac{3 \theta}{2}\right) \\
-\cos \left(\frac{3 \theta}{2}\right) \\
\sin \left(\frac{3 \theta}{2}\right)
\end{array}\right)\right] \\
& +\frac{K_{I I}}{4 \sqrt{2 \pi r}} \times\left[\left(\begin{array}{c}
-3 \sin \left(\frac{\theta}{2}\right) \\
-5 \sin \left(\frac{\theta}{2}\right) \\
\cos \left(\frac{\theta}{2}\right)
\end{array}\right)\right. \\
& \left.+3\left(\begin{array}{c}
-\sin \left(\frac{3 \theta}{2}\right) \\
\sin \left(\frac{3 \theta}{2}\right) \\
\cos \left(\frac{3 \theta}{2}\right)
\end{array}\right)\right]
\end{aligned}
$$

In (2) $K_{I}$ and $K_{I I}$ are the stress intensity factors according to the linear elastic fracture mechanics.

By observing that the circumferential stress $\sigma_{\theta \theta}$ at the direction of crack extension is a principal stress the shear stress $\tau_{r \theta}$ along that direction vanishes. It can be easily shown that the above criterion for the crack growth direction is the solution of the following equation:

$$
K_{I} \sin \theta+K_{I I}(3 \cos \theta-1)=0 .
$$

For the calculation of the stress $\sigma_{\theta \theta}$ from (2) a critical distance $r_{0}$ measured from the crack tip must be introduced. The concept of a core region surrounding the crack tip has been proposed by Sih [18-20]. The idea is that the continuum mechanics solution, as well as experimental measurement, stops at a distance $r_{0}$ from the crack tip. The distance $r_{0}$ serves as a scale size of analysis at the continuum level. This criterion has been widely used because of its simplicity. Moreover, it has been supported by many experimental observations. The application of this criterion can be found from the works by several authors and in particular by Ayatollahi and coworkers who extended the approach to sharp and blunt Vnotches considering not only the singular terms but also the nonsingular ones [8-12].

\section{Sih's Criterion}

The concept of "core region" surrounding the crack tip was proposed in [18-20]. The main idea is that the continuum mechanics stops short at a distance from the crack tip, providing the concept of the radius of the core region. The strain energy density factor $S$ was defined as the product of the strain energy density by a critical distance from the point of singularity. Failure was thought of as controlled by a critical value $S_{c}$, whereas the direction of crack propagation was determined by imposing a minimum condition on $S$. The theory was extended to employ the total strain energy density near the notch tip, and the point of reference was chosen to be the location on the surface of the notch where the maximum tangential stress occurs. It is worth mentioning that Sih's criterion is a pointwise criterion. The material element is always kept at a finite distance from the crack or the notch tip outside the "core region" where the inhomogeneity of the material due to microcracks, dislocations, and grain boundaries precludes an accurate analytical solution. The theory can account for yielding and fracture and is applicable also to ductile materials. Depending on the local stress state, the radius of the core region may or may not coincide with the critical ligament $r_{c}$ that corresponds to the onset of unstable crack extension. The ligament $r_{c}$ depends on the fracture toughness $K_{I C}$, the yield stress $\sigma_{y}$, Poisson's ratio $\nu$, and, finally, on the ratio between dilatational and distortional components of the strain energy density. The direction of $\sigma_{\max }$ determines maximum distortion, while $\sigma_{\min }$ relates to dilatation. Distortion is associated with yielding; dilatation tends to be associated with the creation of free surfaces or fracture and occurs along the line of expected crack extension.

A critical value of strain energy density function $(d W / d V)_{c}$ has been extensively used since 1965, when first the ratio $(d W / d V)_{c}$ was determined experimentally for various engineering materials by using plain and notched specimens. The deformation energy required for crack initiation in a unit volume of material is called Absorbed Specific Fracture Energy (ASFE) and its links with the critical value of $J_{c}$ and the critical factor $S_{c}$ were widely discussed. This topic was deeply considered in [21-23] where it was shown that $(d W / d V)_{c}$ is equivalent to $S_{c} / r$, with $S_{c}$ being the critical strain energy density factor and the radius vector $r$ the location of failure. Since distributions of the absorbed 
specific energy $W$ in notched specimens are not uniform, it was assumed that the specimen cracks as soon as a precise energy amount has been absorbed by the small plastic zone at the root of the notch. If the notch is sufficiently sharp, specific energy due to the elastic deformation is small enough to be neglected as an initial approximation. While measurements of the energy in an infinitely small element are not possible, they can be approximated with sufficient accuracy by calculating the fracture energy over the entire fractured cross section of an unnotched tensile specimen.

Dealing with pointed V-notches the volume energy density factor $S$ was defined and applied as an extension of the method proposed for the crack case. Potential sites of fracture initiation were assessed and the rate change of volume with surface $\Delta V / \Delta A$ was accurately evaluated by using numerical models showing that the local variation of this parameter should be kept smaller than the global average of $\Delta V / \Delta A$ in the system to assure the reliability of the numerical results. Moreover, the critical strain energy density factor, $S_{c}$, was plotted as a function of the notch opening angle both for symmetrical and skew-symmetrical loadings [20].

More precisely Sih wrote $S$ as follows [18-20]:

$$
S=a_{11}(\theta) K_{I}^{2}+2 a_{12}(\theta) K_{I} K_{I I}+a_{22}(\theta) K_{I I}^{2},
$$

where $K_{I}$ and $K_{I I}$ are the stress intensity factors of Linear Elastic Fracture Mechanics and $a_{11}, a_{12}, a_{22}$ are the angular functions depending on Poisson's ratio $v$ and the shear modulus; $G$ is related to Young's modulus $E$ as $E=2 G(1+\nu)$. These functions are

$$
\begin{gathered}
a_{11}=\frac{1}{16 G}[(1+\cos (\theta))(\kappa-\cos (\theta))], \\
a_{12}=\frac{1}{16 G} \sin \theta[2 \cos (\theta)-(\kappa-1)], \\
a_{22}=\frac{1}{16 G}[(\kappa+1)(1-\cos \theta)+(1+\cos \theta)(3 \cos \theta-1)] .
\end{gathered}
$$

Here $\kappa$ takes the value $(3-4 \nu)$ for plane strain and $(3-v) /(1+v)$ for plane stress conditions.

Sih wrote that "the initial crack growth takes place in the direction along which the strain energy density factor $S$ possesses a stationary (minimum) value"; that is,

$$
\frac{\partial S}{\partial \theta}=0
$$

As stated by Sih himself, the $S$-criterion requires no calculation on the energy release rate and thus possesses the inherent advantage of being able to treat all mixed mode problems for the first time. Unlike the conventional Griffith's theory which measures only the amplitude of local stresses, the fundamental parameter $S$ is direction-sensitive. The difference between Griffith's parameter and $S$ is analogous to the difference between a scalar and a vector. Sih's criterion has been applied prevalently to cracks and sharp notches. However, worth mentioning is also the application to two particular notches: elliptic holes and hyperbolic notches [19].
The extension to other notch shapes is possible, with $S$ being a unifying parameter. This is the main advantage of the present approach.

\section{Neuber's Fictitious Notch Rounding (FNR) Approach}

According to Neuber [1-3], the theoretical maximum notch stress is not the effective parameter able to control static or fatigue strength in the case of pointed or sharp notches. The notch stress has to be averaged over a short distance normal to the notch edge. Materials have a specific "elementary" volume, which results in a specific "structural support length." The support effect can be described by averaging the maximum notch stresses in a small material volume (microsupport length $\rho^{*}$ ) at the notch root (with radius $\rho$ ). The averaged stress may be expressed by the maximum stress of a corresponding notch of an enlarged, fictitious radius, with the enlargement being expressed by the material parameter $\rho^{*}$ modified by the microsupport factor $s$ which expresses the influence of multiaxiality and the strength criterion. The length $\rho^{*}$ exclusively depends on the microstructural material conditions, for example, expressed by the yield limit $\sigma_{Y}$ in the high-cycle fatigue range (Neuber [1-3]) or by the fracture toughness $K_{I c}$ and the local fracture stress $\sigma_{m c}$ according to $\rho^{*}=(2 / \pi)\left(K_{I c} / \sigma_{m c}\right)^{2}$ in static loading.

The relation $\rho^{*}=(1 / 2 \pi)\left(K_{I c} / \sigma_{Y}\right)^{2}$ with yield limit $\sigma_{Y}$ (approximately equal to the ultimate strength in the case of brittle fracture) given by Neuber [1-3] is conformed with the "critical distance approach."

The fictitious notch rounding concept and its mathematical foundation originate from a time in which the importance of the notch stresses for strength assessments became clear resulting in a demand to determine these stresses either experimentally or theoretically. In order to fully appreciate Neuber's contribution, it is necessary to consider the microsupport concept within the context of the notch stress theory in general which was an amazing achievement at that time and has remained so up to today.

Following Neuber's concept, Radaj [4-6] proposed to predict the high-cycle fatigue strength of welded joints (toe and root failures) based on fictitious notch rounding. The worst case assessment for low-strength steels introduced $\rho=$ $0 \mathrm{~mm}, s=2.5$, and $\rho^{*}=0.4 \mathrm{~mm}$. This procedure was proven to be generally applicable to welded joints in structural steels and aluminium alloys. It has become a standardised procedure within the IIW design recommendations.

With the aim to make clear the main points of the Neuber's approach only mode I loading is considered here. When the notch radius is very small, or tends to zero in the worst case condition, the theoretical stress concentration factor $K_{t}$ reaches very high values and brittle or fatigue failure of the component can no longer be described by the theoretical peak value of the notch stress. Obviously, not the theoretical peak stress but the stress averaged over a material element of finite size can be assumed as decisive for local failure when considering sharp rounded or pointed notches, that is inclusive of corner notches and cracks. 
The microstructural material element at the notch tip is characterised by the material-dependent microstructural support length $\rho^{*}$. A rough first approximation from experimental results states that $\rho^{*} \approx 0.1 \mathrm{~mm}$ for low-strength steels and aluminium alloys in fatigue. Stress averaging is performed over this length solving the notch problem and integrating the theoretical notch stresses $\sigma_{\text {th }}$ over $\rho^{*}$ :

$$
\bar{\sigma}=\frac{1}{\rho^{*}} \int_{x_{0}}^{x_{0}+\rho^{*}} \sigma_{\mathrm{th}} d x .
$$

In order to simplify this procedure, Figure 2(a), Neuber [1-3] has introduced the concept of fictitious notch rounding. The averaged notch stress $\bar{\sigma}$ can directly be determined by analysing a notch with fictitiously enlarged notch radius $\rho_{f}$, Figure 2(b):

$$
\rho_{f}=\rho+s \rho^{*} .
$$

Here, $\rho$ is the actual notch radius and $s$ is the support factor which depends on the multiaxiality of the stress state and the applied failure criterion, $s=2.0-3.0$ in tension loading and $s=1.0$ in out-of-plane shear loading according to Neuber [1$3]$. The factor $s$ results from performing the averaging process for different loading conditions and equivalent stresses.

The method given by Neuber for determining the fictitious notch radius $\rho_{f}$ (and therefrom the support factor $s$ ) consists of the following steps:

(i) to describe the relevant (equivalent) stress $\sigma$ (or $\tau$ ) in the bisector (that is the assumed crack path) by the closed-form expressions for the sharp rounded Vnotch (without microstructural support);

(ii) to determine the peak stress $\bar{\sigma}$ (or $\bar{\tau}$ ) averaged over the microstructural support length $\rho^{*}$ (other closed-form expressions);

(iii) to equate the expression of $\bar{\sigma}$ (or $\bar{\tau}$ ) for finite $\rho^{*}$ with that of $\bar{\sigma}$ (or $\bar{\tau}$ ) for $\rho^{*}=0$, in order to derive the fictitious radius $\rho_{f}$ dependent on $\rho, \rho^{*}$, and $2 \alpha$ by numerical procedures;

(iv) to derive the factor $s=\left(\rho_{f}-\rho\right) / \rho^{*}$ which is also dependent on $\rho, \rho^{*}$, and $2 \alpha$ in general.

Some lacks and controversies remained in Neuber's approach. In particular it has to be noted that the proposal given in the in-plane shear loading was completely neglected and the values of $s$ were given without any reference to the notch opening angle, while a strong influence of this angle was originally stated by Neuber (at least for sharp notches) considering the normal stress criterion both for tension and torsion loading. Also in the more recent edition of Neuber's book [3], the influence of the notch opening angle has disappeared and a single value of $s$ seems to be proposed to describe fictitious notch rounding for any notch opening angle. The values of $s$ given by Neuber for tension (and bending) loading are referring to the plane stress conditions of flat specimens and to (approximately) plane strain condition of axis-symmetric specimens with a circumferential notch. Another missing point was to provide the blunt notch solution taking into account the influence of other notch geometrical parameters besides the notch radius. In conclusion, some intriguing doubts remain with respect to the application of the approach, in particular when dealing with sharp notches with arbitrary notch opening angles. It has to be also underlined that although Neuber always spoke of "elementary volume" his method is based on an integration on a line and the contribution of the notch flanks is not fully considered in the computation.

With the aim to address the main open points of Neuber's work, the FNR approach was applied to V-notches (see Figure 2) subjected to pure mode I taking advantage of some analytical solutions reported in the literature. The factor $s$, which quantifies the multiaxiality effect, was found to be highly dependent on the notch opening angle $2 \alpha$. Table 1 summarizes the values of $s$ under mode 1 loading and different failure criteria as summarized in a recent review (see [38] and references therein).

Only taking into account the variability of the opening angle, a sound correspondence was found between the theoretical stress concentration factors $K_{t}\left(\rho_{f}\right)$ (evaluated at the fictitiously rounded notch) and the effective stress concentration factors $\bar{K}_{t}$ (obtained by integrating the relevant stress over the distance $\rho^{*}$ in the bisector line of the pointed $\mathrm{V}$ notch).

\section{Cohesive Zone Model Applied to Notches}

The cohesive model was first proposed in the 1960s by Dugdale [39] and Barenblatt [40] from a theoretical point of view to eliminate the stress singularity at the tip of the crack. In the 1970s, Hillerborg [41] generalised the cohesive zone $(\mathrm{CZM})$ model to explain fracture process where no initial macroscopic crack existed. This last generalization is the starting point for applying this model to notches. A review of this model, together with some improvements, was made in a recent paper by Elices and coworkers [24].

The criterion states that a cohesive crack initiates at the point where the maximum principal stress $\sigma_{11}$ first reaches a critical value, being termed the cohesive strength $f_{t}$. This cohesive crack initiates in the direction perpendicular to the maximum principal stress. After its formation, the cohesive crack opens while transferring stress from one face to the other one. The transferred stress strictly depends on the crack opening displacement. For a monotonic load and local mode I, the stress transferred $\sigma$ is normal to the crack faces and is a unique function of the crack opening displacement $w$. The material function linking $\sigma$ and $w$ is termed the softening function (see Figure 3).

The material behaviour is usually characterised by the constitutive equations of the bulk material and the softening function. As shown in [25-28] the bulk material behaviour can, in many cases, be assumed linearly elastic and the softening function a rectangular law (see Figure 4).

The rectangular softening function has been successfully employed for PMMA at $-60^{\circ} \mathrm{C}$ where fracture assessment of notches in mode I was the prime concern [28]. This softening curve is the simplest one and depends only on two parameters: the cohesive stress $f_{t}$ and the fracture energy 


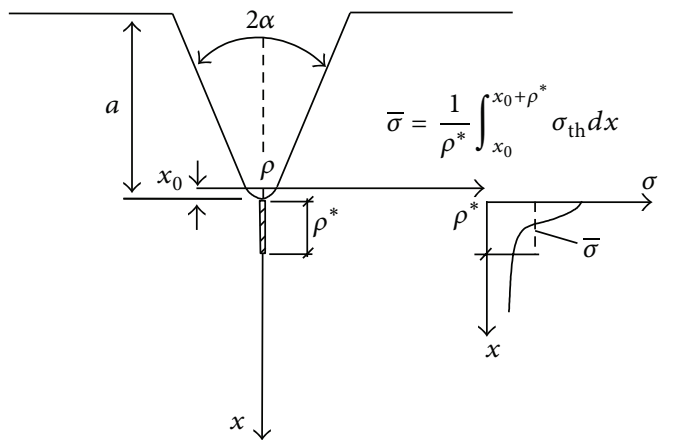

(a)

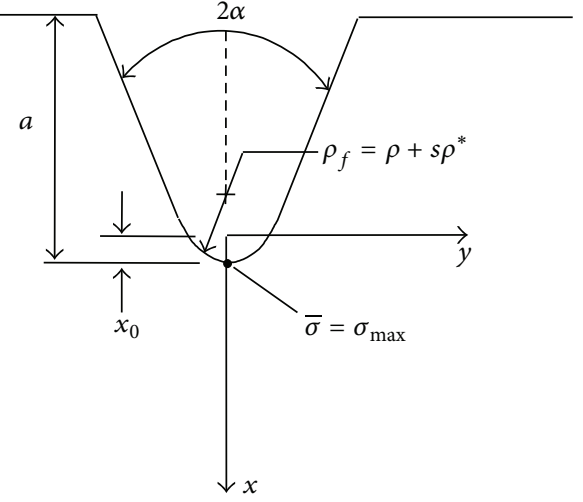

(b)

Figure 2: Application of the notch rounding approach to mode I loading.

TABLE 1: Microstructural support factor $s$ (plateau values) of tension-loaded V-notches for different failure criteria under plane stress and plane strain conditions.

\begin{tabular}{lccccc}
\hline $2 \alpha$ & Normal stress & von Mises plane stress & von Mises plane strain & Beltrami plane stress & Beltrami plane strain \\
\hline $0^{\circ}$ & 2.00 & 2.50 & 2.90 & 2.30 & 2.42 \\
$90^{\circ}$ & 2.81 & 3.37 & 3.80 & 3.14 & 3.28 \\
$120^{\circ}$ & 3.67 & 4.32 & 4.84 & 4.06 & 4.24 \\
$135^{\circ}$ & 4.56 & 5.33 & 5.94 & 5.02 & 5.22 \\
$150^{\circ}$ & 6.38 & 7.41 & 8.20 & 6.99 & 7.25 \\
\hline
\end{tabular}

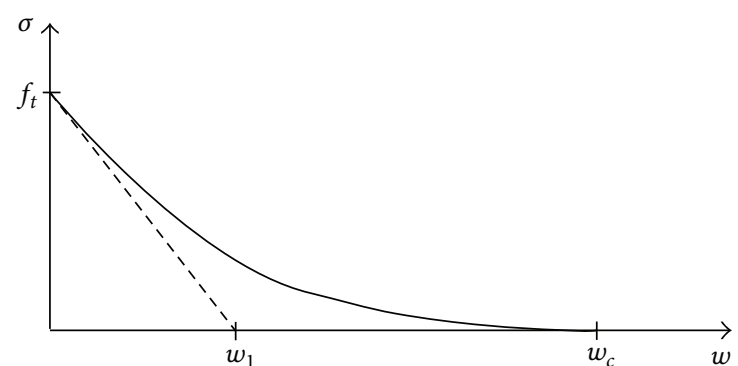

FIGURE 3: Cohesive softening function and initial linear approximation.

$G_{F}$. There, the cohesive strength $f_{t}$ was assumed as equal to the tensile strength measured at $-60^{\circ} \mathrm{C}$ from unnotched specimens, while the fracture energy was calculated from the knowledge of the fracture toughness and

$$
G_{F}=\frac{K_{I C}^{2}}{E^{\prime}}
$$

where $E^{\prime}=E /\left(1-v^{2}\right)$.

In mode I loading, the geometry and loading are symmetrical to the symmetry plane of the notch. This has the advantage of knowing, a priori, the crack path as well as the plane on which the cohesive process zone develops and allows us to model only half of the geometry. The cohesive process zone can be modelled on this plane as a mixed boundary condition by stipulating a relationship between stresses and displacements given by the softening function.

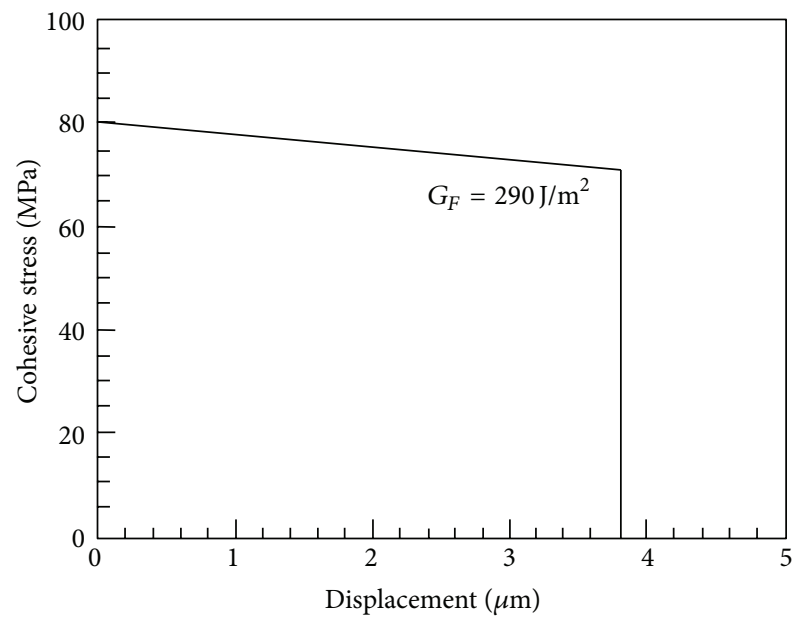

FIgURE 4: Typical softening function for PMMA.

In mixed mode loading the problem is more complex, given that the fracture path is initially unknown. This problem could be overcome by using the local mode I approach, with the hypothesis that the cohesive crack initiates ahead of the notch in the point where the principal stress reaches its maximum value. It could then be analysed by placing close to this point, perpendicular to the notch edge, a band of cohesive elements, where the behaviour is defined by the softening function in a similar manner to mode I. To improve slightly this procedure, a triangle of special elements can be placed 


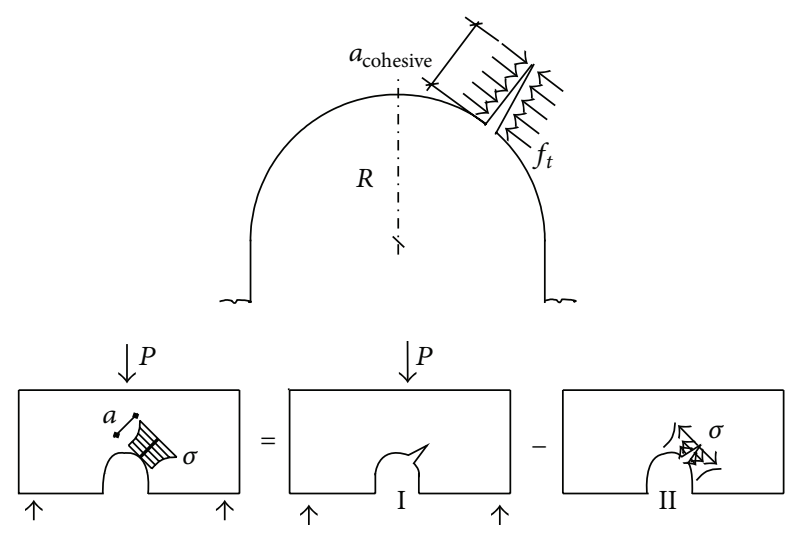

FIGURE 5: Cohesive crack and superposition diagram.

near the critical point, allowing a possible curvature in the initial path of the cohesive crack.

A freeware finite element code (COFE) has been developed at the Department of Materials Science at the Universidad Politécnica de Madrid by Planas and Sancho [42]. The local mode I hypothesis is employed to determine the initial position of the cohesive crack: the point at the notch boundary where the main elastic principal stress is at a maximum. After a first linear elastic calculation to reach such a point, an isosceles triangle is commonly placed over it with the height of the triangle perpendicular to the notch to simulate the cohesive crack. The size of the element decreases near the critical point on the notch up to $4 \mu \mathrm{m}$. Special cohesive elements are placed into the triangular area, using the embedded crack technique. Bulk elements are conventional linear triangular plane strain elements. Calculations are usually performed controlling the displacement of the node at the cohesive crack initial point.

The procedure predicts the maximum load when the maximum displacement between the lips of the cohesive crack reaches the critical value, $w_{c}$, equal to the fracture energy divided by the cohesive stress $f_{t}$. When the problem is formulated analytically and an actual crack is considered, two equations govern fracture: the maximum displacement among the cohesive crack lips and the fact that the stresses are finite near the cohesive crack. Thus, the stress intensity factor must be equal to zero; that is,

$$
\begin{gathered}
w_{c}=\frac{G_{F}}{f_{t}}, \\
K_{I}=0 .
\end{gathered}
$$

Using the superposition principle, the initial state can be analysed as a combination of two auxiliary states, shown in Figure 5: a loaded notched sample, having a crack without transferring stresses and a notched sample without external loads and with a crack loaded by cohesive forces. By introducing this concept into $((10),(11))$ one reaches at the analytical formulation that permits to solve the problem. A general description of these two equations and the detailed procedure can be found in [43].

\section{The Averaged Strain Energy Density Criterion}

Different from Sih's criterion, which is a pointwise criterion, the averaged Strain Energy Density (SED) method as formalized for sharp [29] and blunt notches [30] is reminiscent of Neuber's concept of elementary volume as well as of the local mode I concept proposed by Erdogan and Sih [7]. The averaged SED method condenses together the advantages to be an energy-based criterion with those tied to the definition to a material-dependent structural volume.

With the aim of clarifying the bases of the syntheses presented in this paper both in relation to static failure of brittle or quasi-brittle materials and the fatigue strength of notched components and welded joints, the framework of the SED approach will be presented in detail in the next sections.

The SED approach is based on the idea that under prevailing tensile stresses failure occurs when the strain energy density averaged over a given control volume reaches a critical value, $\bar{W}=W_{c}$, where $W_{c}$ depends on the material. If the material behaviour is ideally brittle, then $W_{c}$ can be evaluated by using simply the conventional ultimate tensile strength $\sigma_{t}$, so that $W_{c}=\sigma_{t}^{2} / 2 E$. In principle $W_{c}$ as determined from uniaxial tests cannot be considered independent of the loading mode. Under compression, for example, the critical value of $W_{c}$ is surely different from the critical value under tension.

Often unnotched specimens exhibit a nonlinear behaviour, whereas the behaviour of notched specimens remains linear. Under these circumstances the stress $\sigma_{t}$ should be substituted by the maximum normal stress existing at the edge at the moment preceding the cracking.

In plane problems, the control volume becomes a circle or a circular sector with a radius $R_{0}$ in the case of cracks or pointed V-notches in mode I or mixed, I + II, mode loading (Figures 6(a), and 6(b)). A useful expression for the radius $R_{0}$ surrounding the control volume has been provided for the crack case under plane strain and plane stress conditions [34, 35]:

$$
\begin{gathered}
R_{0}=\frac{(1+\nu)(5-8 v)}{4 \pi}\left(\frac{K_{I C}}{\sigma_{t}}\right)^{2} \text { plane strain, } \\
R_{0}=\frac{(5-3 \nu)}{4 \pi}\left(\frac{K_{C}}{\sigma_{t}}\right)^{2} \text { plane stress. }
\end{gathered}
$$

In the presence of a notch root radius equal to zero it is possible to determine the total strain energy over the area of radius $R_{0}$ and then the mean value of the elastic SED referred to the area $\Omega$. The final relationship under pure mode I loading and sharp notches is

$$
\bar{W}_{1}=\frac{I_{1}}{4 E \lambda_{1}(\pi-\alpha)}\left(\frac{K_{1}}{R_{0}{ }^{1-\lambda_{1}}}\right)^{2},
$$

where $\lambda_{1}$ is Williams' mode I eigenvalue and $K_{1}$ is the corresponding notch stress intensity factor. The parameter $I_{1}$ is different under plane stress and plane strain conditions and is provided in previous references as a function of the notch opening angle and Poisson's ratio $[34,35]$. 


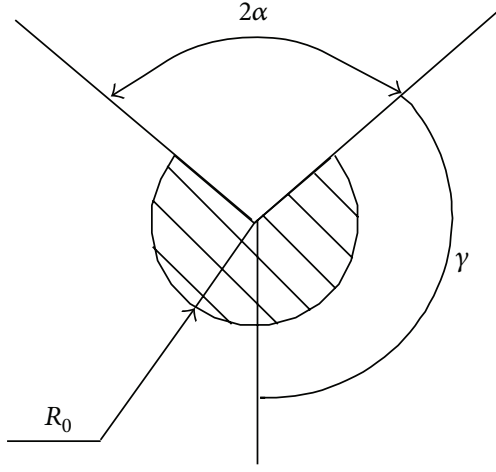

(a)

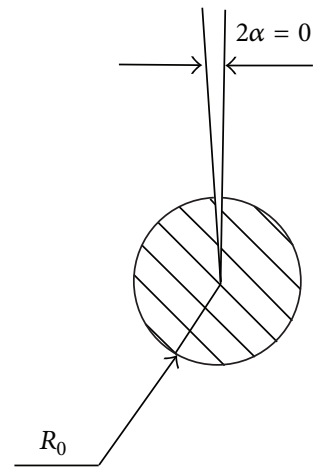

(b)

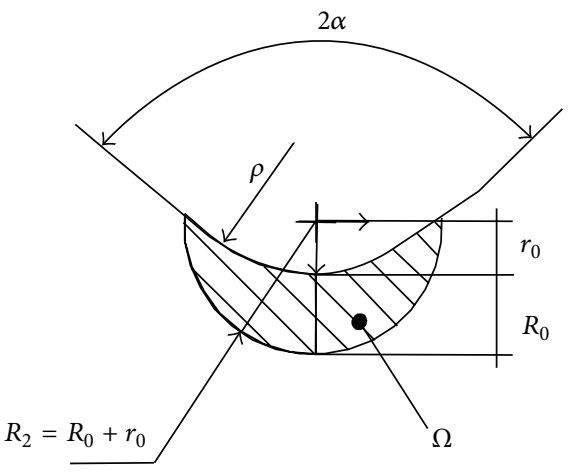

(c)

Figure 6: Critical volume (area) for sharp V-notch (a), crack (b), and blunt V-notch (c) under mode I loading. Distance $r_{0}=\rho \times(\pi-2 \alpha) /(2 \pi-$ $2 \alpha)$.

Equation (13) can be extended to pointed V-notches under a different combination of mode I + II + III loadings $[29,32]$ as a function of the corresponding notch stress intensity factors $K_{1}, K_{2}$, and $K_{3}$ and of the angular functions $e_{1}, e_{2}$, and $e_{3}[34,35]$. Consider

$$
\bar{W}=\frac{e_{1}}{E}\left[\frac{K_{1}}{R_{0}{ }^{1-\lambda_{1}}}\right]^{2}+\frac{e_{2}}{E}\left[\frac{K_{2}}{R_{0}{ }^{1-\lambda_{2}}}\right]^{2}+\frac{e_{3}}{E}\left[\frac{K_{3}}{R_{0}{ }^{1-\lambda_{3}}}\right]^{2} .
$$

In the presence of rounded V-notches it is possible to link the SED to the maximum principal stress at the notch tip. When the area embraces the semicircular edge of the notch (and not its rectilinear flanks), the mean value of SED can be expressed in the following form [30]:

$$
\bar{W}_{1}=F(2 \alpha) \times H\left(2 \alpha, \frac{R_{0}}{\rho}\right) \times \frac{\sigma_{\text {tip }}^{2}}{E},
$$

where $F(2 \alpha)$ depends on the notch opening angle. $H$ is summarised in [30] as a function of opening angles and the Poisson's ratio.

The proposal of mode I dominance was suggested first by Erdogan and Sih [7] when dealing with cracked plates under plane loading and transverse shear, where the crack grows in the direction almost perpendicular to the maximum tangential stress in radial direction from its tip. By testing plexiglass plates weakened by a crack under pure shear loading, Erdogan and Sih showed that the fracture angle varied around 70 degrees with very small scatter. That angle was in agreement with the theoretical value (70.5 degrees) obtained in the skew-symmetric case.

In the case of sharp V-notches two different eigenvalues characterize the in-plane stress field under mode I and under mode II loading and this fact does not allow determining an equivalent NSIF governing the scale effect. The mode I dominance can be directly taken into account by using Sih's parameter $S$ (which the product of the strain energy density and a convenient distance from the point of singularity) or the SED concept over a material-dependent control volume.

In the case of blunt notches under mixed mode loading the maximum elastic stress is out of the notch bisector line

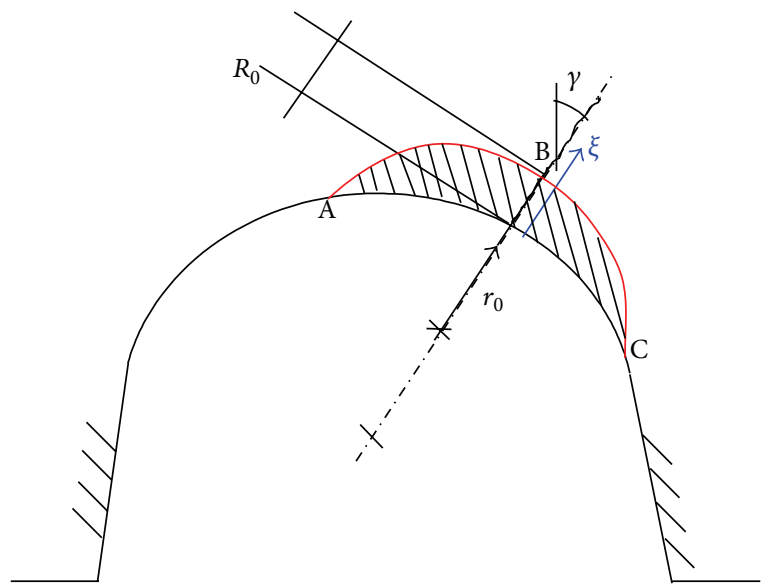

FIGURE 7: V-notch under mixed mode loading.

and its position varies along the notch edge as a function of mode I to mode II stress distributions. This makes the problem of brittle fracture from blunt V-notches more complex than under mode I. In parallel the number of experimental data reported in the literature is quite limited, in particular with reference to prevalent mode II loading conditions, and this makes it difficult to accurately check the different theoretical formulations.

Dealing with blunt $\mathrm{U}$ - and V-notches the concept of equivalent local mode I, although not exact in principle, can be seen as an accurate engineering approximation [44, 45]. In particular the SED was generalised from mode I to mixed mode $(\mathrm{I}+\mathrm{II})$, under the hypothesis of an equivalent local mode I along the normal line to the notch edge, at a point where the principal stress reaches its maximum value. The approach was used to assess rupture loads of U-notched components made of PMMA and tested at $-60^{\circ} \mathrm{C}$ under mixed mode loading $[44,45]$.

According to the coordinate system shown in Figure 7, the stress component $\sigma_{\theta \theta}$ normalised to its maximum value occurring along the notch edge is plotted in Figure 8 as a function of the normalised distance $\xi / \rho$. The inclined path 


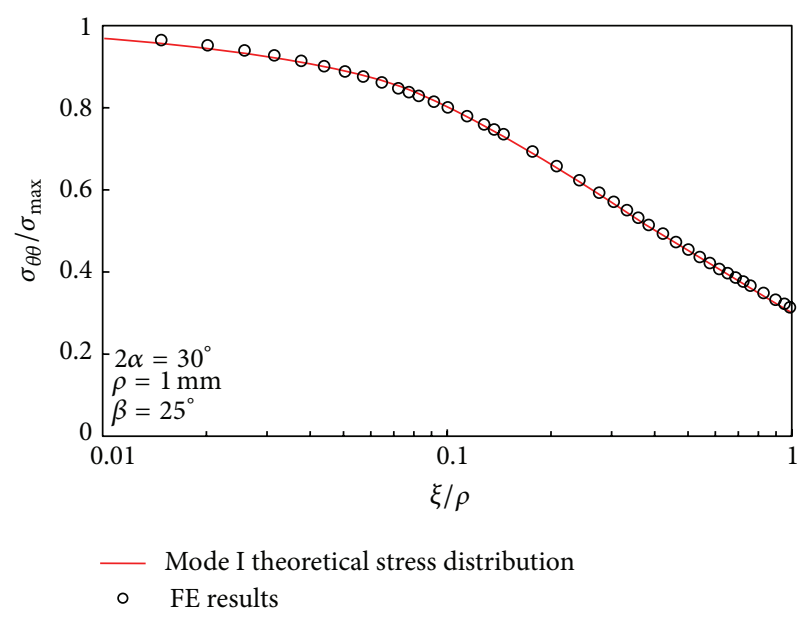

FIGURE 8: Proof of equivalent local mode I.

is perpendicular to the notch edge and starts from the point of the maximum of $\sigma_{\theta \theta}$ stress component along the notch profile. The finite element results are compared with the mode I theoretical solution. The agreement is satisfactory under prevalent mode II, independent of the notch radius. In parallel, the shear stress component has been verified to be zero, as it happens along the notch bisector under mode I loading. This observation leads to the conclusion that under mixed mode loading the line normal to the notch edge and starting from the point of maximum principal stress behaves as a virtual bisector line under pure mode I, confirming the applicability of the equivalent local mode I concept.

The SED can be also easily determined, once defined the control volume, by using a commercial finite element program. In fact, as opposed to the direct evaluation of the stress-based parameters, which needs very refined meshes, the mean value of the elastic SED on the control volume can be determined with high accuracy by using coarse meshes [46]. Very refined meshes are necessary to directly determine the NSIFs from the local stress distributions. Refined meshes are not necessary when the aim of the finite element analysis is to determine the mean value of the local strain energy density on a control volume surrounding the points of stress singularity. The SED in fact can be derived directly from nodal displacements, so that also coarse meshes are able to give sufficiently accurate values for it. As widely described in [35] the SED approach has been recently applied for the fracture assessment of different materials showing the capability of the approach to predict the fracture and fatigue assessments of different kind of materials as well as a large variety of geometries.

\section{Conclusions}

Brittle failure of components weakened by cracks or sharp and blunt V-notches is a topic of active research. It is attractive for all researchers who face the problem of brittle and quasi-brittle fracture of materials under different loading conditions. It involves a large number of applications in different engineering fields.

In this paper some criteria for fracture assessment are reviewed, briefly discussing their assumptions. Several representative criteria are included in the review quoting some recent references where the reader can find more details for the direct application of each considered criterion. However, the list of approaches discussed in this paper is not complete and limited to those criteria more familiar to the author's background and research activity.

\section{Conflict of Interests}

The author declares that there is no conflict of interests regarding the publication of this paper.

\section{References}

[1] H. Neuber, Kerbspannungslehre, Springer, Berlin, Germany, 2nd edition, 1958.

[2] H. Neuber, "Über die berücksichtigung der spannungskonzentration bei festigkeitsberechnungen," Konstruktion, vol. 20, no. 7, pp. 245-251, 1968.

[3] H. Neuber, Kerbspannungslehre, Springer, Berlin, Germany, 3rd edition, 1985.

[4] D. Radaj, Design and Analysis of Fatigue Resistant Welded Structures, Abington, Cambridge, UK, 1990.

[5] D. Radaj, C. M. Sonsino, and W. Fricke, Fatigue Assessment of Welded Joints by Local Approaches, Woodhead Publishing, Cambridge, UK, 2006.

[6] D. Radaj and M. Vormwald, Advanced Methods of Fatigue Assessment, Springer, Heidelberg, Germany, 2013.

[7] F. Erdogan and C. G. Sih, "On the crack extension in plates under plane loading and transverse shear," Journal of Basic Engineering, vol. 85, no. 4, pp. 519-525, 1963.

[8] D. J. Smith, M. R. Ayatollahi, and M. J. Pavier, "The role of Tstress in brittle fracture for linear elastic materials under mixedmode loading," Fatigue \& Fracture of Engineering Materials and Structures, vol. 24, no. 2, pp. 137-150, 2001.

[9] M. R. Ayatollahi and M. R. M. Aliha, "Cracked Brazilian disc specimen subjected to mode II deformation," Engineering Fracture Mechanics, vol. 72, no. 4, pp. 493-503, 2005.

[10] M. R. Ayatollahi, M. R. M. Aliha, and H. Saghafi, "An improved semi-circular bend specimen for investigating mixed mode brittle fracture," Engineering Fracture Mechanics, vol. 78, no. 1, pp. 110-123, 2011.

[11] M. R. Ayatollahi and A. R. Torabi, "Tensile fracture in notched polycrystalline graphite specimens," Carbon, vol. 48, no. 8, pp. 2255-2265, 2010.

[12] M. R. Ayatollahi and A. R. Torabi, "Failure assessment of notched polycrystalline graphite under tensile-shear loading," Materials Science and Engineering A, vol. 528, no. 18, pp. 56855695, 2011.

[13] L. Susmel and D. Taylor, "A simplified approach to apply the theory of critical distances to notched components under torsional fatigue loading," International Journal of Fatigue, vol. 28, no. 4, pp. 417-430, 2006.

[14] L. Susmel and D. Taylor, "On the use of the theory of critical distances to predict static failures in ductile metallic materials 
containing different geometrical features," Engineering Fracture Mechanics, vol. 75, no. 15, pp. 4410-4421, 2008.

[15] L. Susmel and D. Taylor, "The theory of critical distances to predict static strength of notched brittle components subjected to mixed-mode loading," Engineering Fracture Mechanics, vol. 75, no. 3-4, pp. 534-550, 2008.

[16] L. Susmel, "The theory of critical distances: a review of its applications in fatigue," Engineering Fracture Mechanics, vol. 75, no. 7, pp. 1706-1724, 2008.

[17] L. Susmel and D. Taylor, "The theory of critical distances to estimate finite lifetime of notched components subjected to constant and variable amplitude torsional loading," Engineering Fracture Mechanics, vol. 98, pp. 64-79, 2013.

[18] G. C. Sih, "Strain-energy-density factor applied to mixed mode crack problems," International Journal of Fracture, vol. 10, no. 3 , pp. 305-321, 1974.

[19] G. C. Sih, Mechanics of Fracture Initiation and Propagation: Surface and Volume Energy Density Applied as Failure Criterion, Kluwer Academic, Dodrecht, The Netherlands, 1991.

[20] G. C. Sih and J. W. Ho, "Sharp notch fracture strength characterized by critical energy density," Theoretical and Applied Fracture Mechanics, vol. 16, no. 3, pp. 179-214, 1991.

[21] L. F. Gillemot, "Brittle fracture of welded materials," in Proceedings of the 2nd Commonwealth Welding Conference, pp. 353-358, London, UK, 1965.

[22] L. F. Gillemot, "Criterion of crack initiation and spreading," Engineering Fracture Mechanics, vol. 8, no. 1, pp. 239-253, 1976.

[23] L. F. Gillemot, E. Czoboly, and I. Havas, "Fracture mechanics applications of absorbed specific fracture energy: notch and unnotched specimens," Theoretical and Applied Fracture Mechanics, vol. 4, no. 1, pp. 39-45, 1985.

[24] M. Elices, G. V. Guinea, F. J. Gómez, and J. Planas, "The cohesive zone model: advantages, limitations and challenges," Engineering Fracture Mechanics, vol. 69, no. 2, pp. 137-163, 2002.

[25] F. J. Gómez and M. Elices, "Fracture of components with Vshaped notches," Engineering Fracture Mechanics, vol. 70, no. 14, pp. 1913-1927, 2003.

[26] F. J. Gómez and M. Elices, "A fracture criterion for sharp Vnotched samples," International Journal of Fracture, vol. 123, no. 3-4, pp. 163-175, 2003.

[27] F. J. Gómez and M. Elices, "A fracture criterion for blunted Vnotched samples," International Journal of Fracture, vol. 127, no. 3, pp. 239-264, 2004.

[28] F. J. Gómez, M. Elices, and J. Planas, "The cohesive crack concept: application to PMMA at $-60^{\circ} \mathrm{C}$," Engineering Fracture Mechanics, vol. 72, no. 8, pp. 1268-1285, 2005.

[29] P. Lazzarin and R. Zambardi, "A finite-volume-energy based approach to predict the static and fatigue behavior of components with sharp V-shaped notches," International Journal of Fracture, vol. 112, no. 3, pp. 275-298, 2001.

[30] P. Lazzarin and F. Berto, "Some expressions for the strain energy in a finite volume surrounding the root of blunt V-notches," International Journal of Fracture, vol. 135, no. 1-4, pp. 161-185, 2005.

[31] P. Lazzarin, T. Lassen, and P. Livieri, "A notch stress intensity approach applied to fatigue life predictions of welded joints with different local toe geometry," Fatigue \& Fracture of Engineering Materials and Structures, vol. 26, no. 1, pp. 49-58, 2003.

[32] P. Lazzarin, C. M. Sonsino, and R. Zambardi, "A notch stress intensity approach to assess the multiaxial fatigue strength of welded tube-to-flange joints subjected to combined loadings,"
Fatigue \& Fracture of Engineering Materials and Structures, vol. 27, no. 2, pp. 127-140, 2004.

[33] P. Livieri and P. Lazzarin, "Fatigue strength of steel and aluminium welded joints based on generalised stress intensity factors and local strain energy values," International Journal of Fracture, vol. 133, no. 3, pp. 247-278, 2005.

[34] F. Berto and P. Lazzarin, "A review of the volume-based strain energy density approach applied to $\mathrm{V}$-notches and welded structures," Theoretical and Applied Fracture Mechanics, vol. 52, no. 3, pp. 183-194, 2009.

[35] F. Berto and P. Lazzarin, "Recent developments in brittle and quasi-brittle failure assessment of engineering materials by means of local approaches," Materials Science and Engineering: $R$, vol. 75, pp. 1-48, 2014.

[36] F. Berto, P. Lazzarin, and M. R. Ayatollahi, "Brittle fracture of sharp and blunt V-notches in isostatic graphite under torsion loading," Carbon, vol. 63, pp. 101-116, 2013.

[37] F. Berto, D. Cendon, P. Lazzarin, and M. Elices, "Fracture behaviour of notched round bars made of PMMA subjected to torsion at $-60^{\circ} \mathrm{C}$, Engineering Fracture Mechanics, vol. 102, pp. 271-287, 2013.

[38] D. Radaj, P. Lazzarin, and F. Berto, "Generalised Neuber concept of fictitious notch rounding," International Journal of Fatigue, vol. 51, pp. 105-115, 2013.

[39] D. S. Dugdale, "Yielding of steel sheets containing slits," Journal of the Mechanics and Physics of Solids, vol. 8, no. 2, pp. 100-104, 1960.

[40] G. I. Barenblatt, "The mathematical theory of equilibrium of cracks in brittle fracture," Advances in Applied Mechanics, vol. 7, pp. 55-129, 1962.

[41] A. Hillerborg, M. Modéer, and P. E. Petersson, "Analysis of crack formation and crack growth in concrete by means of fracture mechanics and finite elements," Cement and Concrete Research, vol. 6, no. 6, pp. 773-781, 1976.

[42] J. Planas and J. M. Sancho, "Computational Orientated Finite Elements, COFE," Tech. Rep. JP0501, Departamento de Ciencia de los Materiales, Universidad Politécnica de Madrid, Madrid, Spain, 2007.

[43] Z. P. Bažant and J. Planas, Fracture and Size Effect in Concrete and Other Quasibrittle Materials, CRC Press, Boca Raton, Fla, USA, 1998.

[44] F. J. Gómez, M. Elices, F. Berto, and P. Lazzarin, "Local strain energy to assess the static failure of U-notches in plates under mixed mode loading," International Journal of Fracture, vol. 145, no. 1, pp. 29-45, 2007.

[45] F. Berto, P. Lazzarin, F. J. Gómez, and M. Elices, "Fracture assessment of U-notches under mixed mode loading: two procedures based on the "equivalent local mode I" concept," International Journal of Fracture, vol. 148, no. 4, pp. 415-433, 2007.

[46] P. Lazzarin, F. Berto, and M. Zappalorto, "Rapid calculations of notch stress intensity factors based on averaged strain energy density from coarse meshes: theoretical bases and applications," International Journal of Fatigue, vol. 32, no. 10, pp. 1559-1567, 2010. 

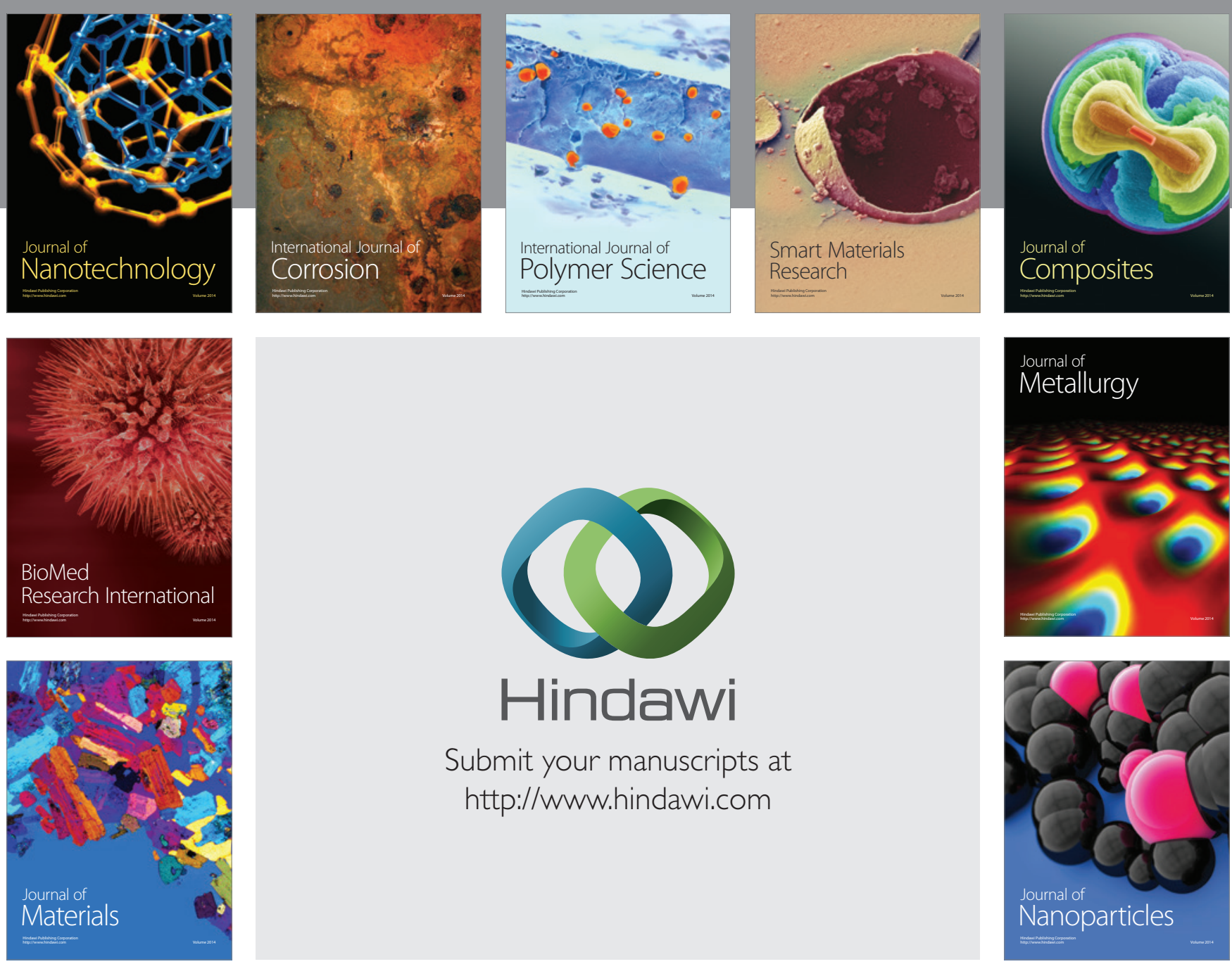

Submit your manuscripts at http://www.hindawi.com
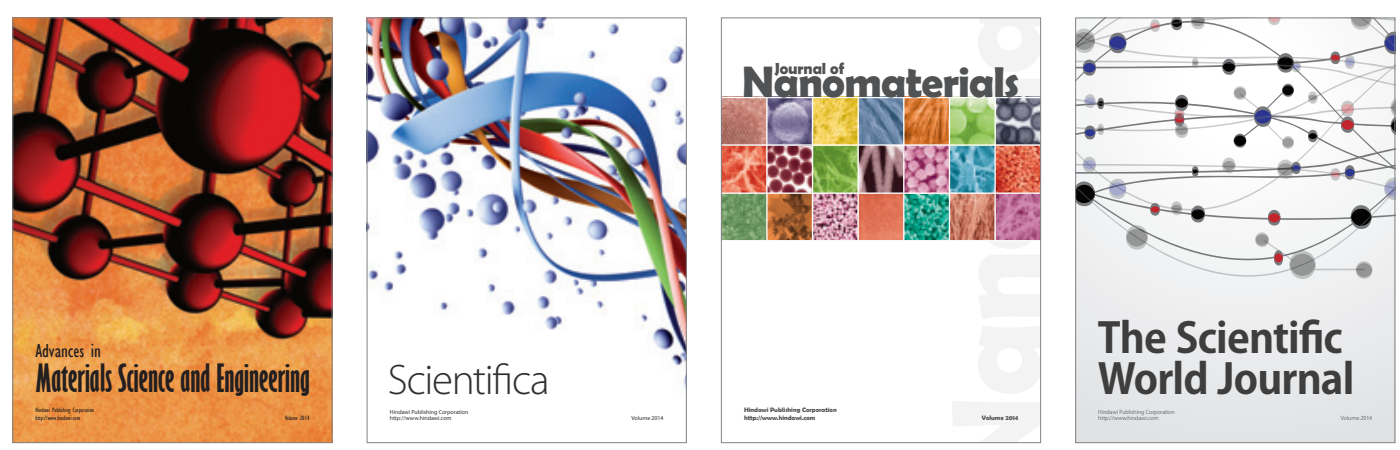

\section{The Scientific World Journal}
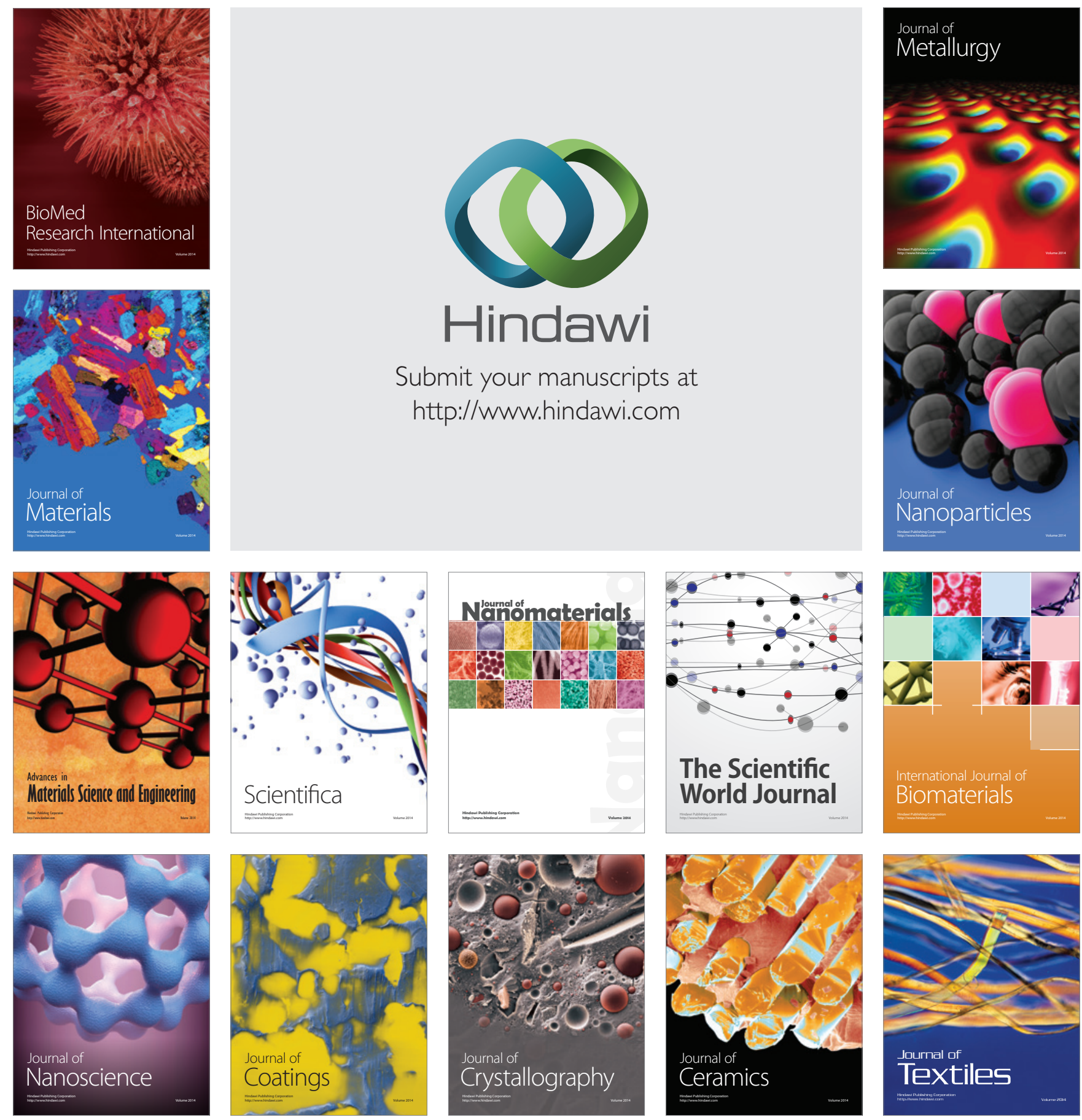